\title{
Pairings and Copairings in the Category of Topological Spaces
}

By

\author{
Nobuyuki ODA*
}

\begin{abstract}
We prove a theorem which gives a relation between the pairings of homotopy sets induced by pairings and copairings of topological spaces. We obtain many results on commutativity of elements of homotopy sets as immediate consequences of the theorem. As a further application of the theorem, we prove a generalization of a theorem of Sugawara on the existence of inverse element in the homotopy set when the target space is a Hopf space. We also prove the dual result which is a generalization of a theorem of Hilton, Mislin and Roitberg.
\end{abstract}

\section{§1. Introduction}

We work in the category of G-spaces (namely, G-equivariant spaces) with base point $*$ which is fixed under the $G$-action. So we denote simply by $f: X \rightarrow Y$ a $G$-map. We simply write $f \simeq g$ when $f$ is $G$-homotopic to $g$. The group $G$ is a topological group throughout the paper.

We call a continuous map $\mu: X \times Y \rightarrow Z$ a pairing with axes $f: X \rightarrow Z$ and $g: Y \rightarrow Z$ when $\mu \mid X \times\{*\} \simeq f$ and $\mu \mid\{*\} \times Y \simeq g$ (Definition 2.1). Dually we define a copairing $\theta: A \rightarrow B \vee C$ with coaxes $h: A \rightarrow B$ and $r: A \rightarrow C$ (Definition 2.4.)

In a previous paper [13], we studied some properties of axes of pairings and coaxes of copairings of topological spaces. We also obtained fundamental results on the homotopy sets of the axes and coaxes. On the other hand, pairings and copairings of topological spaces induce pairings of homotopy sets. In this paper we study some of the properties of these induced pairings.

We now state them more precisely. Given a pairing $\mu: X \times Y \rightarrow Z$, we can define a $G$-map $\alpha+\beta: A \rightarrow Z$ for any $G$-maps $\alpha: A \rightarrow X$ and $\beta: A \rightarrow Y$ by the formula (2.2) in $\S 2$. Dually, given a copairing $\theta: A \rightarrow B \vee C$, we can define a $G$-map $\alpha \dot{+} \beta: A \rightarrow Z$ for any $G$-maps $\alpha: B \rightarrow Z$ and $\beta: C \rightarrow Z$ by the formula

Communicated by M. Kashiwara, June 25, 1990.

1991 Mathematics Subject Classification: 55P91,

* Department of Applied Mathematics, Faculty of Science, Fukuoka University, 8-19-1, Nanakuma, Jonanku, Fukuoka 814-01, Japan. 
(2.5) in $\S 2$. Let $[A, Z]$ be the $G$-homotopy set of all the $G$-maps from $A$ to $Z$. Then we have the following main result of this paper.

Theorem 2.7. Let $\alpha: B \rightarrow X, \beta: C \rightarrow X, \gamma: B \rightarrow Y$ and $\delta: C \rightarrow Y$ be $G$-maps. Let $\mu: X \times Y \rightarrow Z$ be a pairing with axes $f: X \rightarrow Z$ and $g: Y \rightarrow Z$, and $\theta: A \rightarrow B \vee C$ a copairing with coaxes $h: A \rightarrow B$ and $r: A \rightarrow C$. Then the following relations hold in $[A, Z]$.

(1) $(\alpha \dot{+} \beta)+(\gamma+\delta)=(\alpha+\gamma) \dot{+}(\beta+\delta)$.

(2) $h^{*}(\boldsymbol{\alpha})+r^{*}(\boldsymbol{\delta})=f_{*}(\boldsymbol{\alpha}) \dot{+} g_{*}(\boldsymbol{\delta})$.

(3) $r^{*}(\beta)+h^{*}(\gamma)=g_{*}(\gamma)+f_{*}(\beta)$.

In Theorem 2.7, the formulae (2) and (3) are special cases of the formula (1). The result $(2)$ says that two pairing + and $\dot{+}$ coincide when composed with the axes and coaxes, and hence (3) says that the pairings are commutative when composed with the axes and coaxes. So, this theorem is a generalization of the well-known result that the homotopy set $[A, Z]$ is an abelian group when $A$ is a co-Hopf space and $Z$ is a Hopf space, as we see by setting $h \simeq r \simeq 1_{A}$ and $f \simeq g \simeq 1_{z}$ in Theorem 2.7. We prove the theorem by generalizing the method of Hilton [6].

In $\S 3$ we show that various important results concerning commutativity of elements in homotopy set are direct consequences of Theorem 2.7. These are properties of cyclic maps, cocyclic maps and filtrations of homotopy sets given by, for example, CW decomposition of the source space or Postnikov decomposition of the target space.

In $\S 4$ we prove a theorem which generalizes a result of Sugawara as an application of Theorem 2.7. Sugawara $[15,16]$ proved a theorem which asserts that each elements of $[A, X]$ has a left inverse element and a right inverse element under some conditions when $X$ is a Hopf space. Our first result is stated in the following theorem.

Theorem 4.1. Let $A$ be a Hausdorff G-CW complex and $X$ and $Y$ G-0-connected Hausdorff $G-C W$ complexes. Suppose that $Y$ acts on $X$ by a G-map $\mu: X \times Y$ $\rightarrow X$. Then for each element $\alpha$ of $[A, X]$ and each element $\beta$ of $[A, Y]$, there exists a unique element $\gamma$ of $[A, X]$ such that $\gamma+\beta=\alpha$.

In the proof of the above theorem, we use Theorem 2.7 to know the fact that the pairing + induced by $\mu: X \times Y \rightarrow Z$ coincides with the ordinary sum + induced by the co-Hopf structure of $n$-sphere $S^{n}$ in homotopy group $\pi_{n}(Z)$. This enables us to handle the pairing + as the usual sum + in the homotopy group $\pi_{n}(Z)$

In Theorem 4.2, we prove the dual result of Theorem 4.1. It is a gener- 
alization of a result of Hilton, Mislin and Roitberg [7].

The author would like to express his thanks to Professor M. Mimura for valuable comments and to Professor T. Matumoto for valuable information on G-CW complexes.

\section{$\S 2$. Pairings and Copairings}

Let $\Delta_{X}: X \rightarrow X \times X$ be the diagonal map defined by $\Delta_{X}(x)=(x, x)$ for any element $x$ of $X$ and $\nabla_{X}: X \vee X \rightarrow X$ the folding map defined by $\nabla_{X}(x, *)=x=$ $\nabla_{X}(*, x)$ for any element $x$ of $X$. We consider the wedge sum (or the one point union) $X \vee Y$ of $X$ and $Y$ as the subspace of the product space $X \times Y$ with the inclusion map $j: X \vee Y \rightarrow X \times Y$.

Definition 2.1. We call a $G$-map $\mu: X \times Y \rightarrow Z$ a pairing with axes $f: X \rightarrow Z$ and $g: Y \rightarrow Z$, if the diagram

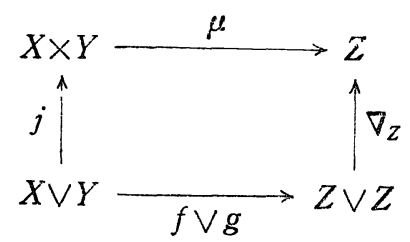

is $G$-homotopy commutative.

Remark. A Hopf space $X$ is a topological space with a continuous multiplication $m: X \times X \rightarrow X$ with a two-sided unit, namely,

$$
m \mid X \vee X \simeq \nabla_{X} \circ\left(1_{X} \vee 1_{X}\right): X \vee X \rightarrow X
$$

for the folding map $\nabla_{X}: X \vee X \rightarrow X$. To generalize the concept of the Gottlieb group [5], Varadarajan [18] introduced a map $F: X \times A \rightarrow X$ with a cyclic map $f: A \rightarrow X$, namely,

$$
F \mid X \vee A \simeq \nabla_{X} \circ\left(1_{X} \vee f\right): X \vee A \rightarrow X .
$$

More generally, Stasheff [14] considered a map $f: X \times Y \rightarrow Z$ with axes $g: X \rightarrow Z$ and $h: Y \rightarrow Z$, namely,

$$
f \mid X \vee Y \simeq \nabla_{Z} \circ(g \vee h): X \vee Y \rightarrow Z .
$$

This is a pairing in Definition 2.1. Stasheff [14] called a map $f: X \times Y \rightarrow Z$ an axial map when the axes are inclusion maps $g: X \subset Z$ and $h: Y \subset Z$. There are dual concepts of the above definitions $[3,6,18,21]$.

Now, suppose that we are given a pairing $\mu: X \times Y \rightarrow Z$. Then we can 
define a map $\alpha+\beta: A \rightarrow Z$ for any $G$-maps $\alpha: A \rightarrow X$ and $\beta: A \rightarrow Y$ by

$$
\alpha+\beta=\mu \circ(\alpha \times \beta) \circ \Delta_{A} .
$$

This defines a pairing of homotopy sets

$$
+:[A, X] \times[A, Y] \rightarrow[A, Z] .
$$

Now, we denote by $*: X \rightarrow Y$ the constant map.

Proposition 2.3. If $\mu: X \times Y \rightarrow Z$ is a pairing with axes $f: X \rightarrow Z$ and $g: Y \rightarrow Z$, then

$$
\alpha+*=f_{*}(\alpha) \text { and } *+\beta=g_{*}(\beta)
$$

in $[A, Z]$ for any $G$-maps $\alpha: A \rightarrow X$ and $\beta: A \rightarrow Y$.

Proof. Let us suppose that $\beta=*$. Then we have

$$
(\alpha \times *) \circ \Delta_{A}=j \circ j_{1} \circ \alpha: A \rightarrow X \times Y
$$

for the inclusion map $j_{1}: X \rightarrow X \vee Y$ defined by $j_{1}(x)=(x, *)$ for any element $x$ of $X$. It follows that

$$
\alpha+*=\mu \circ(\alpha \times *) \circ \Delta_{A}=\mu \circ j \circ j_{1} \circ \alpha \simeq f \circ \alpha=f_{*}(\alpha) .
$$

Similarly we have the latter result.

Definition 2.4. We call a $G$-map $\theta: A \rightarrow B \vee C$ a copairing with coaxes $h: A \rightarrow B$ and $r: A \rightarrow C$, if the diagram

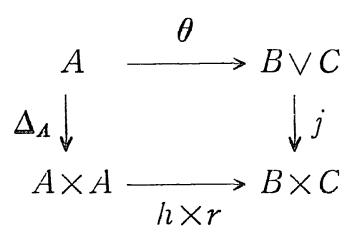

is $G$-homotopy commutative.

Given a copairing $\theta: A \rightarrow B \vee C$, we define a $G$-map $\alpha+\beta: A \rightarrow Z$ for any $G$-maps $\alpha: B \rightarrow Z$ and $\beta: C \rightarrow Z$ by

$$
\alpha+\beta=\nabla_{Z} \circ(\alpha \vee \beta) \circ \theta .
$$

This defines a pairing of homotopy sets

$$
+:[B, Z] \times[C, Z] \longrightarrow[A, Z] \text {. }
$$

Proposition 2.6. If $\theta: A \rightarrow B \vee C$ is a copairing with coaxes $h: A \rightarrow B$ and $r: A \rightarrow C$, then

$$
\alpha \dot{+} *=h^{*}(\alpha) \text { and } * \dot{+} \beta=r^{*}(\beta)
$$


in $[A, Z]$ for any $G$-maps $\alpha: B \rightarrow Z$ and $\beta: C \rightarrow Z$.

Proof. Let us suppose that $\beta=*$. Then we have

$$
\nabla_{Z} \circ(\alpha \vee *)=\alpha \circ q_{1}: B \vee C \rightarrow Z
$$

for the projection map $q_{1}: B \vee C \rightarrow B$ defined by $q_{1}(x, *)=x$ for any element $x$ of $B$ and $q_{1}(*, y)=*$ for any element $y$ of $C$. It follows that

$$
\alpha \dot{+} *=\nabla_{Z} \circ(\alpha \vee *) \circ \theta=\alpha \circ q_{1} \circ \theta \simeq \alpha \circ h=h^{*}(\alpha) .
$$

Similarly we have the latter result.

Theorem 2.7. Let $\alpha: B \rightarrow X, \beta: C \rightarrow X, \gamma: B \rightarrow Y$ and $\delta: C \rightarrow Y$ be $G$-maps. Let $\mu: X \times Y \rightarrow Z$ be a pairing with axes $f: X \rightarrow Z$ and $g: Y \rightarrow Z$, and $\theta: A \rightarrow B \vee C$ a copairing with coaxes $h: A \rightarrow B$ and $r: A \rightarrow C$. Then the following relations hold in $[A, Z]$.

(1) $(\alpha \dot{+} \beta)+(\gamma \dot{+} \delta)=(\alpha+\gamma) \dot{+}(\beta+\delta)$.

(2) $h *(\alpha)+r^{*}(\delta)=f_{*}(\boldsymbol{\alpha}) \dot{+} g_{*}(\boldsymbol{\delta})$.

(3) $r^{*}(\beta)+h^{*}(\gamma)=g_{*}(\gamma) \dot{+} f_{*}(\beta)$.

Proof. We first prove (1). By Definitions 2.1 and 2.4, we have

$$
\begin{aligned}
(\alpha \dot{+} \beta)+(\gamma \dot{+} \delta) & =\mu \circ\left\{\left(\nabla_{X} \circ(\alpha \vee \beta) \circ \theta\right) \times\left(\nabla_{Y} \circ(\gamma \vee \delta) \circ \theta\right)\right\} \circ \Delta_{A} \\
& =\mu \circ\left(\nabla_{X} \times \nabla_{Y}\right) \circ\{(\alpha \vee \beta) \times(\gamma \vee \delta)\} \circ(\theta \times \theta) \circ \Delta_{A} \\
& =\mu \circ\left(\nabla_{X} \times \nabla_{Y}\right) \circ\{(\alpha \vee \beta) \times(\gamma \vee \delta)\} \circ \Delta_{B \vee C} \circ \theta \\
& =\mu \circ \nabla_{X \times Y} \circ\{(\alpha \times \gamma) \vee(\beta \times \delta)\} \circ\left(\Delta_{B} \vee \Delta_{C}\right) \circ \theta \\
& =\nabla_{Z} \circ(\mu \vee \mu) \circ\{(\alpha \times \gamma) \vee(\beta \times \delta)\} \circ\left(\Delta_{B} \vee \Delta_{C}\right) \circ \theta \\
& =\nabla_{Z} \circ\left\{\left(\mu \circ(\alpha \times \gamma) \circ \Delta_{B}\right) \vee\left(\mu \circ(\beta \times \delta) \circ \Delta_{C}\right)\right\} \circ \theta \\
& =(\alpha+\gamma)+(\beta+\delta)
\end{aligned}
$$

This completes the proof of (1). To prove (2) and (3), set in (1) $\beta=\gamma=*$ for (2), and $\alpha=\delta=*$ for (3), then we have the results by Propositions 2.3 and 2.6.

Proposition 2.8. Let $\mu: X \times Y \rightarrow Z$ be a pairing with axes $f: X \rightarrow Z$ and $g: Y \rightarrow Z$, and $\theta: A \rightarrow B \vee C$ be a copairing with coaxes $h: A \rightarrow B$ and $r: A \rightarrow C$. Then the following results hold.

(1) If $X=Y$ and $f \simeq g$, then

$$
h^{*}(\alpha)+r^{*}(\beta)=r^{*}(\beta)+h^{*}(\alpha)
$$

in $[A, Z]$ for any elements $\alpha: B \rightarrow X$ and $\beta: C \rightarrow X$.

(2) If $B=C$ and $h \simeq r$, then 


$$
f_{*}(\boldsymbol{\beta}) \dot{+} g_{*}(\boldsymbol{\delta})=g_{*}(\boldsymbol{\delta}) \dot{+} f_{*}(\boldsymbol{\beta})
$$

in $[A, Z]$ for any elements $\beta: B \rightarrow X$ and $\delta: B \rightarrow Y$.

Proof. (1) Putting $\delta=\beta$ and $\gamma=\alpha$ in (2) and (3) of Theorem 2.7 respectively, we have

$$
h^{*}(\alpha)+r^{*}(\beta)=f_{*}(\alpha) \dot{+} g_{*}(\beta) \text { and } r^{*}(\beta)+h^{*}(\alpha)=g_{*}(\alpha) \dot{+} f_{*}(\beta) .
$$

Since $f \simeq g$ by our assumption, we have $h^{*}(\alpha)+r^{*}(\beta)=r^{*}(\beta)+h^{*}(\alpha)$.

(2) Putting $\alpha=\beta$ and $\gamma=\delta$ in (2) and (3) of Theorem 2.7 respectively, we have

$$
h^{*}(\beta)+r^{*}(\delta)=f_{*}(\beta)+g_{*}(\delta) \text { and } r^{*}(\beta)+h^{*}(\delta)=g_{*}(\delta)+f_{*}(\beta) .
$$

Since $h \simeq r$ by our assumption, we have $f_{*}(\beta) \dot{+} g_{*}(\delta)=g_{*}(\delta) \dot{+} f_{*}(\beta)$.

\section{$\S 3$. Various Results on Commutativity}

A $G$-space $X$ is called a $H o p f G$-space $[1,17]$ when $X=Y=Z$ and $f \simeq g \simeq 1_{X}$ in Definition 2.1 .

A $G$-space $A$ is called a co-Hopf $G$-space [2] when $A=B=C$ and $h \simeq r \simeq 1_{A}$ in Definition 2.4.

We have the following well-known result $[6,21]$ as an immediate consequence of Theorem 2.7.

Proposition 3.1. Let $A$ be a co-Hopf G-space and $X$ a Hopf $G$-space. Then for any elements $\alpha$ and $\beta$ of $[A, X]$, we have

(1) $\alpha+\beta=\alpha \dot{+} \beta$.

(2) $\alpha+\beta=\beta+\alpha \quad$ where we write $+=+=+$ by (1).

Proof. Set $f \simeq g \simeq 1_{X}$ and $h \simeq r \simeq 1_{A}$ in Theorem 2.7(2)(3), then we have the result.

q.e.d.

We say that a $G$-space $Y$ acts on a $G$-space $X$ from the right when $X=Z$ and $f \simeq 1_{X}$ in Definition 2.1. In this case, $g: Y \rightarrow X$ is called a cyclic map [18].

We say that a $G$-space $C$ coacts on a $G$-space $A$ from the right when $A=B$ and $h \simeq 1_{A}$ in Definition 2.4. In this case, $r: A \rightarrow C$ is called a cocyclic map [18].

The above situations were also studied by Eckmann and Hilton $[3,6]$ as operators and cooperators in connection with fibre sequences and cofibre sequences.

Proposition 3.2. Let $Z$ be a Hopf G-space.

(1) If $\theta: A \rightarrow B \vee C$ is a copairing with coaxes $h: A \rightarrow B$ and $r: A \rightarrow C$, then $\alpha+\beta=h^{*}(\alpha)+r^{*}(\beta)$ and $h^{*}(\alpha)+r^{*}(\beta)=r^{*}(\beta)+h^{*}(\alpha)$ 
in $[A, Z]$ for any elements $\alpha$ of $[B, Z]$ and $\beta$ of $[C, Z]$.

(2) If $C$ coacts on $A$ from the right with the cocyclic map $r: A \rightarrow C$, then

$$
\alpha+\beta=\alpha+r^{*}(\beta) \text { and } \alpha+r^{*}(\beta)=r^{*}(\beta)+\alpha
$$

in $[A, Z]$ for any elements $\alpha$ of $[A, Z]$ and $\beta$ of $[C, Z]$; that is, the image of the cocyclic map

$$
r^{*}:[C, Z] \longrightarrow[A, Z]
$$

is contained in the center of $[A, Z]$.

Proof. (1) By Theorem 2.7(2) (Set $f \simeq g \simeq 1_{Z}$ ) and Proposition 2.8(1).

(2) Since $A=B$ and $h \simeq 1_{A}: A \rightarrow A$, we have the result by (1). q.e.d.

The above Proposition 3.2(2) is a generalization of Corollary 3.10 of Lim [9]. As an example of Proposition 3.2(2), we have the following well-known results. Let $\Sigma X$ be the reduced suspension space and $\Omega X$ the loop space of a $G$-space $X$.

Example 3.3. If

$$
U \stackrel{i}{\longrightarrow} V \stackrel{j}{\longrightarrow} W \stackrel{\delta}{\longrightarrow} \Sigma U \longrightarrow \cdots
$$

is a $G$-cofibre sequence, then we have a $G$-map

$$
\theta: W \longrightarrow W \vee \Sigma U
$$

with the cocyclic map $\delta: W \rightarrow \Sigma U[3,4,6]$. It follows that the image of

$$
\delta^{*}:[\Sigma U, Z] \longrightarrow[W, Z]
$$

is contained in the center of the target object for any Hopf $G$-space $Z$ by Proposition 3.2(2). Therefore the image of

$$
\delta^{*}:[\Sigma U, \Omega Z] \longrightarrow[W, \Omega Z] \quad\left(\text { or }(\Sigma \delta)^{*}:\left[\Sigma^{2} U, Z\right] \longrightarrow[\Sigma W, Z]\right)
$$

is contained in the center of the target group for any $G$-space $Z$.

Proposition 3.4. Let $A$ be a co-Hopf G-space.

(1) If $\mu: X \times Y \rightarrow Z$ is a pairing with axes $f: X \rightarrow Z$ and $g: Y \rightarrow Z$, then

$$
\alpha+\beta=f_{*}(\alpha) \dot{+} g_{*}(\beta) \text { and } f_{*}(\alpha) \dot{+} g_{*}(\beta)=g_{*}(\beta) \dot{+} f_{*}(\alpha)
$$

in $[A, Z]$ for any elements $\alpha$ of $[A, X]$ and $\beta$ of $[A, Y]$.

(2) If $Y$ acts on $Z$ from the right with the cyclic map $g: Y \rightarrow Z$, then

$$
\alpha+\beta=\alpha \dot{+} g_{*}(\beta) \text { and } \alpha \dot{+} g_{*}(\beta)=g_{*}(\beta) \dot{+} \alpha
$$

in $[A, Z]$ for any elements $\alpha$ of $[A, Z]$ and $\beta$ of $\left[A, Y^{*}\right]$; that is, the image of the cyclic map 


$$
g_{*}:[A, Y] \longrightarrow[A, Z]
$$

is conlained in the center of $[A, Z]$.

Proof. (1) By Theorem 2.7(2) (Set $h \simeq r \simeq 1_{A}$ ) and Proposition 2.8(2).

(2) Since $X=Z$ and $f \simeq 1_{Z}: Z \rightarrow Z$, we have the result by (1). q.e.d.

The result of Proposition 3.4(2) is a generalization of Theorem 2 of Hoo [8]. As an example of Proposition 3.4(2), we have the following well-known result.

Example 3.5. Let

$$
\cdots \longrightarrow \stackrel{\stackrel{\partial}{\longrightarrow}}{\longrightarrow} F \stackrel{i}{\longrightarrow} E \stackrel{p}{\longrightarrow} B
$$

be a $G$-fibration sequence. Then there exists a $G$-map

$$
\mu: F \times \Omega B \longrightarrow F
$$

with the cyclic map $\partial: \Omega B \rightarrow F$ by Proposition 11.3 of $[6]$ or $[3,4]$. Then the image of

$$
\partial_{*}:[A, \Omega B] \longrightarrow[A, F]
$$

is contained in the center of the target object for any co-Hopf $G$-space $A$ by Proposition 3.4(2). Therefore the image of

$$
\partial_{*}:[\Sigma A, \Omega B] \longrightarrow[\Sigma A, F] \quad\left(\text { or }(\Omega \partial)_{*}:\left[A, \Omega^{2} B\right] \longrightarrow[A, \Omega F]\right)
$$

is contained in the center of the target group for any $G$-space $A$.

We remark that Theorem 2.7 gives us various formulae in the following cases. (1) $h \simeq 1_{A}$ and $f \simeq 1_{X}$; (2) $r \simeq 1_{A}$ and $f \simeq 1_{X}$; (3) $h \simeq 1_{A}$ and $g \simeq 1_{Y}$; (4) $r \simeq 1_{A}$ and $g \simeq 1_{Y}$.

Proposition 3.6. (1) Let $C$ oact on $A$ from the right $\left(h \simeq 1_{A}\right)$ and $Y$ act on $X$ from the right $\left(f \simeq 1_{X}\right)$. Then

$$
\begin{array}{ll}
\alpha+r^{*}(\boldsymbol{\delta})=\alpha+g_{*}(\boldsymbol{\delta}) & \text { for any } \alpha \text { of }[A, X] \text { and } \delta \text { of }[C, Y] ; \\
r^{*}(\beta)+\gamma=g_{*}(\gamma) \dot{+} \beta & \text { for any } \beta \text { of }[C, X] \text { and } \gamma \text { of }\left[A, Y^{Y}\right] .
\end{array}
$$

(2) Let $B$ coact on $A$ from the left $\left(r \simeq 1_{A}\right)$ and $Y$ act on $X$ from the right $\left(f \simeq 1_{X}\right)$. Then

$$
\begin{array}{ll}
h^{*}(\alpha)+\delta=\alpha+g_{*}(\delta) & \text { for any } \alpha \text { of }[B, X] \text { and } \delta \text { of }[A, Y] ; \\
\beta+h^{*}(\gamma)=g_{*}(\gamma) \dot{+} \beta & \text { for any } \beta \text { of }[A, X] \text { and } \gamma \text { of }[B, Y] .
\end{array}
$$

(3) Let $C$ coact on $A$ from the right $\left(h \simeq 1_{A}\right)$ and $X$ act on $Y$ from the left $\left(g \simeq 1_{Y}\right)$. Then 


$$
\begin{array}{ll}
\alpha+r^{*}(\delta)=f_{*}(\alpha)+\delta & \text { for any } \alpha \text { of }[A, X] \text { and } \delta \text { of }[C, Y] ; \\
r^{*}(\beta)+\gamma=\gamma+f_{*}(\beta) & \text { for any } \beta \text { of }[C, X] \text { and } \gamma \text { of }[A, Y] .
\end{array}
$$

(4) Let $B$ coact on $A$ from the left $\left(r \simeq 1_{A}\right)$ and $X$ act on $Y$ from the left $\left(g \simeq 1_{Y}\right)$. Then

$$
\begin{array}{ll}
h^{*}(\alpha)+\delta=f_{*}(\alpha)+\delta & \text { for any } \alpha \text { of }[B, X] \text { and } \delta \text { of }[A, Y] ; \\
\beta+h^{*}(\gamma)=\gamma+f_{*}(\beta) & \text { for any } \beta \text { of }[A, X] \text { and } \gamma \text { of }[B, Y] .
\end{array}
$$

A $G$-space $Y$ is called a group-like $G$-space $[19,21]$ when it is homotopy associative Hopf $G$-space with a homotopy inverse $\nu: Y \rightarrow Y$, namely, $1_{Y}+\nu \simeq *$ $\simeq \nu+1_{Y}$. Then the homotopy set $[X, Y]$ has a group structure for any space $X$. The inverse $-\left[\int\right]: X \rightarrow Y$ is given by $-[f]=[\nu \circ f]$ for any element $[f]: X \rightarrow Y$.

A $G$-space $X$ is called a cogroup-like G-space [21] when it is homotopy associative co-Hopf $G$-space with a homotopy inverse $\nu: X \rightarrow X$, namely, $1_{X} \dot{+} \nu \simeq$ $* \simeq \nu+1_{X}$. Then the homotopy set $\left[X, I^{r}\right]$ has a group structure for any space $Y$. The inverse $-[f]: X \rightarrow Y$ is given by $\dot{-}[f]=[f \circ \nu]$ for any element $[f]: X \rightarrow Y$.

Let $\Gamma$ be a group. A central chain of $\Gamma$ is a sequence of subgroups

$$
\Gamma=\Gamma_{0} \supset \Gamma_{1} \supset \cdots \supset \Gamma_{n} \supset \cdots
$$

such that $\left[\Gamma, \Gamma_{i}\right] \subset \Gamma_{i+1}$ for all $i$, where $[A, B]$ is the commutator subgroup generated by commutators

$$
\left\{a b a^{-1} b^{-1} \mid a \in A \text { and } b \in B\right\} .
$$

If we are given a filtration

$$
X_{0} \stackrel{i_{0}}{\subset} X_{1} \stackrel{i_{1}}{\complement} \cdots \subset X_{n} \stackrel{i_{n}}{\complement} X_{n+1} \subset \cdots \subset X
$$

of a $G$-space $X$, we define

$$
\Gamma_{i}(X, Y)=\left\{[f]: X \rightarrow Y|f| X_{i} \simeq *\right\} \subset[X, Y] .
$$

The following result is a generalization of Lemma 2.14 of Whitehead [19].

Proposition 3.7. Let $Y$ be a group-like G-space. If $X$ is a G-space with flitration

$$
X_{0} \stackrel{i_{0}}{\subset} X_{1} \stackrel{i_{1}}{\complement} \cdots \subset X_{n} \stackrel{i_{n}}{\complement} X_{n+1} \subset \cdots \subset X
$$

such that there is a cofibration sequence

$$
U_{n} \stackrel{f_{n}}{\longrightarrow} X_{n} \stackrel{i_{n}}{\longrightarrow} X_{n+1} \stackrel{\delta_{n}}{\longrightarrow} \Sigma U_{n} \longrightarrow \cdots
$$


for each $n$, then

$$
\Gamma_{0}(X, Y) \supset \Gamma_{1}(X, Y) \supset \cdots \supset \Gamma_{i}(X, Y) \supset \cdots
$$

is a central chain.

Proof. Let $[f] \in \Gamma_{0}(X, Y)$ and $[g] \in \Gamma_{n}(X, Y)$ be any elements. We have to show that

$$
[f]+[g]-[f]-[g] \in \Gamma_{n+1}(X, Y) .
$$

Consider the exact sequence

$$
\left[\Sigma U_{n}, Y\right] \stackrel{\delta_{n}^{*}}{\longrightarrow}\left[X_{n+1}, Y\right] \stackrel{i_{n}^{*}}{\longrightarrow}\left[X_{n}, Y\right] .
$$

Since $i_{n}^{*}\left(g \mid X_{n+1}\right)=g \mid X_{n} \simeq *$, we have

$$
\left[g \mid X_{n+1}\right] \in \operatorname{Im}\left(\delta_{n}^{*}:\left[\Sigma U_{n}, Y\right] \longrightarrow\left[X_{n+1}, Y\right]\right) .
$$

It follows that $\left[g \mid X_{n+1}\right]$ is contained in the center of $\left[X_{n+1}, Y\right]$ by Example 3.3. Therefore we have

$$
([f]+[g]-[f]-[g]) \mid X_{n+1}=\left[f \mid X_{n+1}\right]+\left[g \mid X_{n+1}\right]-\left[f \mid X_{n+1}\right]-\left[g \mid X_{n+1}\right]=*
$$

in $\left[X_{n+1}, Y\right]$. It follow that $[f]+[g]-[f]-[g] \in \Gamma_{n+1}(X, Y)$.

q.e.d.

If we are given a system

$$
Y \stackrel{q_{n+1}}{\longrightarrow} Y_{n+1} \stackrel{p_{n}}{\longrightarrow} Y_{n} \longrightarrow \cdots \stackrel{p_{1}}{\longrightarrow} Y_{1} \stackrel{p_{0}}{\longrightarrow} Y_{0}
$$

such that $q_{n} \simeq p_{n} \circ q_{n+1}$ for a $G$-space $Y$, we define

$$
\bar{\Gamma}_{i}(X, Y)=\left\{[f]: X \longrightarrow Y \mid q_{i} \circ f \simeq *\right\} \subset[X, Y] .
$$

Proposition 3.8. Let $X$ be a cogroup-like G-space. If $Y$ is a G-space with a system

$$
Y \stackrel{q_{n+1}}{\longrightarrow} Y_{n+1} \stackrel{p_{n}}{\longrightarrow} Y_{n} \longrightarrow \cdots \stackrel{p_{1}}{\longrightarrow} Y_{1} \stackrel{p_{0}}{\longrightarrow} Y_{0}
$$

such that $q_{n} \simeq p_{n} \circ q_{n+1}$ and there is a fibration sequence

for each $n$, then

$$
\cdots \longrightarrow \Omega K_{n} \stackrel{\partial_{n}}{\longrightarrow} Y_{n+1} \stackrel{p_{n}}{\longrightarrow} Y_{n} \stackrel{k_{n}}{\longrightarrow} K_{n}
$$

is a central chain.

$$
\bar{\Gamma}_{0}(X, Y) \supset \bar{\Gamma}_{1}(X, Y) \supset \cdots \supset \bar{\Gamma}_{i}(X, Y) \supset \cdots
$$

Proof. Let $[f] \in \bar{\Gamma}_{0}(X, Y)$ and $[g] \in \bar{\Gamma}_{n}(X, Y)$ be any elements. We have to show that

$$
[f] \dot{+}[g] \div[f] \div[g] \in \bar{\Gamma}_{n+1}(X, Y) .
$$

Consider the exact sequence 


$$
\left[X, \Omega K_{n}\right] \stackrel{\partial_{n *}}{\longrightarrow}\left[X, Y_{n+1}\right] \stackrel{p_{n *}}{\longrightarrow}\left[X, Y_{n}\right] .
$$

Since $q_{n *}([g])=p_{n *}\left\{q_{n+1 *}([g])\right\} \simeq *$, we have

$$
q_{n+1 *}([g]) \in \operatorname{Im}\left(\partial_{n *}:\left[X, \Omega K_{n}\right] \longrightarrow\left[X, Y_{n+1}\right]\right) .
$$

It follows that $q_{n+1 *}([g])$ is contained in the center of $\left[X, Y_{n+1}\right]$ by Example 3.5. Therefore we have

$$
q_{n+1 *}([f] \dot{+}[g] \dot{-}[f] \dot{-}[g])=\left(q_{n+1 *}[f]\right) \dot{+}\left(q_{n+1 *}[g]\right)-\left(q_{n+1 *}[f]\right) \dot{-}\left(q_{n+1 *}[g]\right)=*
$$

in $\left[X_{n+1}, Y\right]$. Thus we have $[f] \dot{+}[g] \div[f] \div[g] \in \bar{\Gamma}_{n+1}(X, Y)$.

\section{§4. A Generalization of a Theorem of Sugawara and its Dual}

In this section we work in the category of Hausdorff $G$-CW complexes.

If $X$ is a Hopf space and $A$ is any space, then the homotopy set $[A, X]$ has a binary operation with a two-sided unit. Sugawara $[15,16]$ proved that each element of $[A, X]$ has a left inverse element and a right inverse element under some conditions. Whitehead $[20]$ has also studied the conditions of the theorem of Sugawara.

If $A$ is a co-Hopf space and $X$ is any space, then the homotopy set $[A, X]$ has a binary operation with a two-sided unit. Hilton, Mislin and Roitberg [7] proved that each element of $[A, X]$ has a left inverse element and a right inverse element under some conditions.

The existence of the inverse elements in the homotopy set $[A, X]$ is analyzed in Chapter 17 of Hilton [6] or Chapter 3 of Whitehead [21].

We now suppose that a $G$-space $Y$ acts on a $G$-space $X$ from the right, namely, $X=Z$ and $f \simeq 1_{X}$ in Definition 2.1. Then we have a homotopy commutative diagram

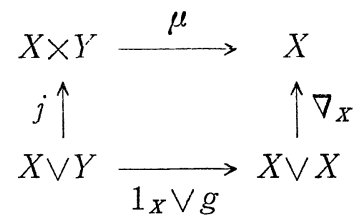

with cyclic map $g: Y \rightarrow X$. Then we have an action

$$
+:[A, X] \times[A, Y] \longrightarrow[A, X]
$$

of $[A, Y]$ on $[A, X]$ defined by

$$
\alpha+\beta=\mu \circ(\alpha \times \beta) \circ \Delta_{A} \in[A, X]
$$

for each element $\alpha$ of $[A, X]$ and each element $\beta$ of $[A, Y]$. In the above situation, we prove Theorem 4.1 which generalizes the result of Sugawara. 
The proof is carried out by generalizing the methods in $[6,15,16,20,21]$.

A $G$-space $X$ is called $G$-n-connected (resp. $G$-simple) if the fixed point set $X^{H}$ is $n$-connected (resp. 0 -connected and simple) for any closed subgroup $H$ of $G$. The references of the $G-C W$ complex are $[1,10,11,12]$. We note that Matumoto [12] has constructed a $G$-CW complex $K_{X}$ and a weak $G$-homotopy equivalence $\rho_{X}: K_{X} \rightarrow X$ for any $G$-space $X$ which satisfies the $T_{1}$-separability condition.

Theorem 4.1. Let $A$ be a Hausdorff G-CW complex and $X$ and $Y$ G-0-connected Hausdorff $G$-CW complexes. Suppose that $Y$ acts on $X$ by a G-map $\mu: X \times Y \rightarrow X$. Then for each element $\alpha$ of $[A, X]$ and each element $\beta$ of $[A, Y]$, there exists a unique element $\gamma$ of $[A, X]$ such that $\gamma+\beta=\alpha$.

Proof. We consider a $G$-map $\phi: X \times Y \rightarrow X \times Y$ defined by $\phi(x, y)=$ $(\mu(x, y), y)$. Then the $G$-map $\phi$ induces a map

(a) $\phi_{*}:[A, X] \times[A, Y] \cong[A, X \times Y] \longrightarrow[A, X \times Y] \cong[A, X] \times[A, Y]$.

We see that

(b)

$$
\phi_{*}(\alpha, \beta)=(\alpha+\beta, \beta)
$$

for any $\alpha$ of $[A, X]$ and any $\beta$ of $[A, Y]$. If $A=G / H^{+} \wedge S^{\imath}$ for $i \geqq 1$, then by Proposition 3.4(2), we have

$$
\alpha+\beta=\alpha+g_{*}(\beta)
$$

where the symbol + means + which is the usual sum induced by the co-Hopf structure of $S^{2}$. Hence the homomorphism

$$
\dot{\phi}_{*}^{H}: \pi_{i}\left((X \times Y)^{H}\right) \longrightarrow \pi_{i}\left((X \times Y)^{I I}\right)
$$

is an isomorphism for any subgroup $H$ of $G$ and for any $i \geqq 1$. Therefore the map $\phi_{*}$ in (a) is an isomorphism for any $G$-CW complex $A$ by Theorem 5.2 of [10] or Proposition 0.4 of [12]. Hence for any pair $(\alpha, \beta)$ of $[A, X] \times[A, Y]$, there exists a unique element $(\gamma, \delta)$ of $[A, X] \times[A, Y]$ such that $\phi_{*}(\gamma, \delta)=$ $(\gamma+\delta, \delta)=(\alpha, \beta)$. It follows that $\gamma+\delta=\alpha$ and $\delta=\beta$, namely, $\gamma+\beta=\alpha$. This completes the proof.

We now consider the dual situation. Let us suppose that a $G$-space $C$ coacts on a $G$-space $A$ from the right, namely, $A=B$ and $h \simeq 1_{A}$ in Definition 2.4. Then we have a homotopy commutative diagram

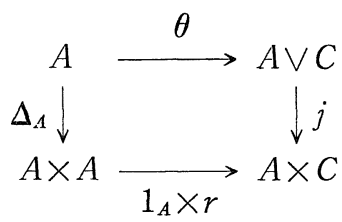


with the cocyclic map $r: A \rightarrow C$. Then we have an action

$$
\dot{+}:[A, X] \times[C, X] \longrightarrow[A, X]
$$

of $[C, X]$ on $[A, X]$ defined by

$$
\alpha \dot{+} \beta=\nabla_{X} \circ(\alpha \vee \beta) \circ \theta \in[A, X]
$$

for any elements $\alpha$ of $[A, X]$ and $\beta$ of $[C, X]$.

We prove now a theorem which generalizes the result of Hilton, Mislin and Roitberg [7]. The proof is done by generalizing their method to the coaction of $G$-spaces in the equivariant category.

Theorem 4.2. Let $A, C$ and $X$ be G-0-connected Hausdorff G-CW complexes. Suppose that $C$ coacts on $A$ by a G-map $\theta: A \rightarrow A \vee C$. Suppose that one of the following conditions is satisfied.

(1) $A$ and $C$ are G-1-connected.

(2) $X$ is G-simple.

Then for each elements $\alpha$ of $[A, X]$ and $\beta$ of $[C, X]$, there exists a unique element $\gamma$ of $[A, X]$ such that $\gamma \dot{+} \beta=\alpha$.

Proof. We define a $G$-map $\phi: A \vee C \rightarrow A \vee C$ by

$$
\phi \mid A=\theta: A \longrightarrow A \vee C \text { and } \phi ! C=i_{2}: C \longrightarrow A \vee C,
$$

where $i_{2}$ is the inclusion map defined by $i_{2}(x)=(*, x)$ for any element $x$ of $C$. We see that

$$
\phi=\left(1_{A} \vee \nabla_{C}\right) \circ\left(\theta \vee 1_{C}\right)
$$

as in [7]. The G-map $\phi$ induces a map

(c) $\phi^{*}:[A, X] \times[C, X] \cong[A \vee C, X] \longrightarrow[A \vee C, X] \cong[A, X] \times[C, X]$.

We see that

$$
\psi^{*}(\alpha, \beta)=(\alpha+\beta, \beta)
$$

for any element $\alpha$ of $[A, X]$ and any element $\beta$ of $[C, X]$.

The same is true when the maps and spaces are restricted to any fixed point sets in (d).

We now consider the conditions (1) and (2) separately.

(1) The induced homomorphism

$$
\left(\phi^{H}\right)_{*}: H_{i}\left((A \vee C)^{H}\right) \longrightarrow H_{i}\left((A \vee C)^{H}\right)
$$

of the integral homology groups of the fixed point set $(A \vee C)^{H}$ is an isomorphism for any subgroup $H$ of $G$ and any $i \geqq 0$, since $\left(\psi^{H}\right)_{*}(\alpha, \beta)=\left(\alpha, r_{*}(\alpha)+\beta\right)$ for any element $\alpha$ of $H_{i}\left(A^{H}\right)$ and $\beta$ of $H_{i}\left(C^{H}\right)$ by the definition of $\psi$, where 
the symbol + means $\dot{+}$ which is the usual sum in the homology groups. If $A$ and $C$ are $G$-1-connected, the isomorphism $\left(\psi^{H}\right)_{*}$ in the homology groups in (e) implies an isomorphism

$$
\left(\phi^{H}\right)_{*}: \pi_{i}\left((A \vee C)^{H}\right) \longrightarrow \pi_{i}\left((A \vee C)^{H}\right)
$$

in the homotopy groups for any subgroup $H$ of $G$ and any $i \geqq 0$ by the Whitehead theorem. It follows that the $G$-map $\psi: A \vee C \rightarrow A \vee C$ is a $G$-homotopy equivalence by the equivariant Whitehead theorem $[1,10,12]$. Then $\psi^{*}$ in (c) is an isomorphism.

(2) Let us substitute the equivariant Eilenberg-MacLane complex $K\left(\underline{\pi}_{n}(X), k\right)$ for $X$ in (c). Then we have a map

$$
\psi^{*}: H_{G}^{k}\left(A \vee C ; \underline{\pi}_{n}(X)\right) \longrightarrow H_{G}^{k}\left(A \vee C ; \underline{\pi}_{n}(X)\right) .
$$

We see that

$$
\psi^{*}(\alpha, \beta)=(\alpha+\beta, \beta)
$$

for any element $\alpha$ of $H_{G}^{k}\left(A: \underline{\pi}_{n}(X)\right)$ and any element $\beta$ of $H_{G}^{k}\left(C ; \underline{\pi}_{n}(X)\right)$ by (d) or the definition of $\phi$. We remark that

$$
\alpha+\beta=\alpha+r^{*}(\beta),
$$

where the symbol + means + which is the usual sum induced by the loop structure of $K\left(\underline{\pi}_{n}(X), k\right)$; it is the result of Proposition 3.2(2). Then the homomorphism $\psi^{*}$ in (f) is an isomorphism for all $k$ and $n$. Considering $\psi: A \vee C(=Y)$ $\rightarrow A \vee C(=Z)$ as an inclusion map, we see

$$
H_{G}^{i}\left(Z, Y ; \underline{\pi}_{\imath}(X)\right)=H_{G}^{i}\left(Z, Y ; \underline{\pi}_{i-1}(X)\right)=0
$$

for all $i$ by the above argument and the long cohomology exact sequences. It follows that $\psi^{*}:[Z, X] \rightarrow[Y, X]$ is an isomorphism by the $G$-obstruction theory $[1,11]$. Then the map $\psi^{*}$ in (c) is an isomorphism.

Now, by the above arguments (1) and (2), we see that for any element $(\alpha, \beta)$ of $[A, X] \times[C, X]$, there exists a unique element $(\gamma, \delta)$ of $[A, X] \times$ $[C, X]$ such that $\phi^{*}(\gamma, \delta)=(\gamma \dot{+} \delta, \delta)=(\alpha, \beta)$. Then we have $\gamma+\delta=\alpha$ and $\delta=\beta$ and hence $\gamma \dot{+} \beta=\alpha$. This completes the proof.

The following result of Corollary 4.3(1) is a generalization of the existence theorem of inversion of Sugawara $[14,16]$.

Corollary 4.3. (1) Let $X$ be a G-0-connected Hausdorff $G$-CW complex and $\mu: X \times X \rightarrow X$ be a pairing with axes $1_{X}: X \rightarrow X$ and some map $g: X \rightarrow X$. Then there exists a map $\nu: X \rightarrow X$ such that $[\nu]+1_{X}=*$ in $[X, X]$.

(2) Let $A$ be a G-simple Hausdorff $G$-CW complex and $\theta: A \rightarrow A \vee A$ be a copairing with coaxes $1_{A}: A \rightarrow A$ and some map $r: A \rightarrow A$. Then there exists a map $\nu: A \rightarrow A$ such that $[\nu]+1_{A}=*$ in $[A, A]$. 
Proof. (1) Set $A=X=Y, \alpha=*$ and $\beta=1_{X}$ in Theorem 4.1, then we have the result.

(2) Set $A=C=X, \alpha=*$ and $\beta=1_{A}$ in Theorem 4.2, then we have the result.

\section{References}

[1] Bredon, G.E., Equivariant cohomology theories, Lecture Notes in Math., 34, Springer-Verlag, Berlin Hidelberg New York, 1967.

[2] Doman, R., On rational co-Hopf G-spaces, Math. Z., 196 (1987), 109-115.

[3] Eckmann, B. and Hilton, P. J., Operators and cooperators in homotopy theory, Math. Ann., 141 (1960), 1-21.

[4] Gania, T., Induced fibrations and cofibrations, Trans. Amer. Math. Soc., 127 (1967), 442-459.

[5] Gottlieb, D. H., A certain subgroup of the fundamental group, Amer. J. Math., 87 (1965), 840-856.

[6] Hilton, P., Homotopy theory and duality, Notes on Mathematics and its Applications, Gordon and Breach, New York London Paris, 1965.

[7] Hilton, P., Mislin, G. and Roitberg, J., On co-H-spaces, Comment. Math. Helv., 53 (1978), 1-14.

[8] Hoo, C.S., Cyclic maps from suspensions to suspensions. Canad. J. Math., 24 (1972), 789-791.

[9] Lim, K.L., Cocyclic maps and coevaluation subgroups, Canad. Math. Bull., 30 (1987), 63-71.

[10] Matumoto. T., On G-CW complexes and a theorem of J.H.C. Whitehead, J. Fac. Sci. Univ. Tokyo, 18 (1971), 363-374.

[11] - Equivariant cohomology theories on G-CW complexes, Osaka J. Math., 10 (1973), 51-68.

[12] - A complement to the theory of G-CW complexes, Japan J. Math., 10 (1984), 353-374.

[13] Oda, N.. The homotopy set of the axes of pairings, Canad. J. Math., 42 (1990), 856-868.

[14] Stasheff, J., On homotopy abelian $H$-spaces, Proc. Cambridge Phil. Soc., 57 (1961), 734-745.

[15] Sugawara, M., On a condition that a space is an $H$-space, Math. J. Okayama Univ., 6 (1957), 109-129.

[16] -, A condition that a space is group-like, Math. J. Okayama Univ., 7 (1957), 123-149.

[17] Triantafillou, G.V., Rationalization of Hopf G-spaces, Math. Z., 182 (1983), 485500.

[18] Varadarajan, K., Generalized Gottlieb groups, J. Indian Math. Soc., 33 (1969), 141164.

[19] Whitehead, G.W., On mapping into group-like spaces, Comment. Math. Helv., 28 (1954), 320-328.

[20] - Note on a theorem of Sugawara, Bol. Soc. Mat. Mexicana, 4 (1959), $33-41$.

[21] - Elements of homotopy theory, Graduate texts in Math., 61, SpringerVerlag, New York Heidelberg Berlin. 1978. 
